MODELING, IDENTIFICATION AND CONTROL, 1983, VOL. 4, NO. 1, 33-45

doi:10.4173/mic.1983.1.3

\title{
Adaptive ship autopilot with wave filter
}

\author{
S. SÆLID $†$ and N. A. JENSSEN $\ddagger \S$
}

Keywords: Adaptive control; wave filter; convergence analysis; autopilot.

This paper is concerned with analysis and design of an adaptive autopilot for ships. The design is based on a low and high frequency model of the vessel motion adequate to ship steering. The low frequency model describes the vessel response to rudder control and slowly varying environmental forces. The high frequency model represents the wave induced oscillatory part of the yaw motion. The models are used in a Kalman filter and the rudder control is computed from linear quadratic theory based on the low frequency part of the vector. This yields a very effective filtering of the wave component of the yaw motion. Proper operation of this filter/controller structure requires knowledge of the vessel model parameters and the dominating wave frequency. The vessel parameters are estimated on line by a recursive prediction error method. In order to reduce the computing requirements, the state estimator is operated using scheduled gains. This results in an easy and robust design. The convergence properties are investigated by using the method of Ljung. The performance is confirmed by simulation experiments.

\section{Introduction}

Traditionally autopilots for ship steering are based on a PID controller. The measured heading signal is compared to the desired heading and the error is used as input to the controller, which activates the rudder servo mechanism. Proper performance of this kind of autopilot requires individual tuning for each installation. In addition the vessel dynamics changes with vessel speed, sea current, load, wind and water depth. This results in bad control behaviour and economic loss due to counter forces induced by cross coupling between yaw rate and sway velocity when the course is changing. By tightening the control loop, the course deviations may be decreased. This, however, results in retarding forces due to the increased rudder motion.

It has been shown by Norrbin (1972) that the average increase in drag due to yawing and rudder motion can be approximately described by

$$
\frac{\Delta D}{D}=\alpha\left[\bar{\psi}^{2}+\lambda \bar{\delta}^{2}\right]
$$

where $D$ is the drag, $\bar{\psi}^{2}$ and $\bar{\delta}^{2}$ are the mean square of the heading error and the rudder angle respectively and $\alpha$ and $\lambda$ are ship dependent parameters.

In recent literature several authors have proposed and analysed different kinds of adaptive autopilots. Some are based on tuning of PID parameters to approximately minimize $\Delta D / D$, others are based on model reference technique. References to these works are given in Källström et al. (1979). Åstrøm and Källström (1976) and Källström et al. (1979) have designed and analysed an autopilot based on a self tuning regulator.

Received 4 October 1982.

† The Norwegian Institute of Technology, Trondheim.

¥ SINTEF, Division of Automatic Control, NTH, Trondheim.

$\S$ Present affiliation, Kongsberg Våpenfabrikk A/S, Maritime Division, Kongsberg. 
The model used in the regulator is of black box type having, e.g. seven unknown parameters to be estimated on line. An alternative approach is to use a model based on physical laws and physically meaningful parameters. This makes it possible to include known mechanisms and to neglect minor effects and parameters $a$ priori.

This paper describes a design and analysis of an adaptive autopilot based on this kind of model. The model has six states and four parameters. The states are estimated by a scheduled gain state estimator and the parameters are estimated by a prediction error method. Feedback from the state estimates are computed partly from optimal control theory.

A main contribution of this paper is the choice of the vessel model, which has two parts. A low-frequency part (LF-part) accounts for the steering dynamics when oscillatory water waves are not present. A high-frequency part (HF-part) models the oscillatory part of the yaw motion due to water waves. Now the autopilot should not try to counteract the HF-motion. Control of this motion would increase the drag both in terms of rudder action and yaw oscillation relative to the water masses. Hence the controller takes feedback only from the LF-part of the motion estimate. In a conventional autopilot, much of the rudder action power is concentrated near the wave frequency (Ohtsu et al. 1979). This is also indicated in a paper written by Källström and Astrom (1981). They found a pair of complex conjugate poles from a steering dynamics identification experiment using real vessel measurements. The present approach should therefore yield better results with respect to increased drag due to autopilot operation.

Section 2 of this paper presents the model used in the autopilot and $\S 3$ describes and analyses the state estimation, the parameter estimation and the control algorithms. A convergence analysis based on the method of Ljung $(1977 \mathrm{~b}, 1981)$ is presented in $\S 4$. Simulation results are shown in $\S 5$ where the adaptive autopilot is tested against a realistic simulator.

\section{Ship steering model}

An HF-model and an LF-model are formulated.

\section{LF-model}

For the present purpose we use the following linearized model as a starting point (Norrbin 1970).

$$
\left[\begin{array}{l}
\dot{r} \\
\dot{v}
\end{array}\right]=u_{0}\left[\begin{array}{cc}
a_{11}^{\prime} & a_{12}^{\prime} \\
a_{21}^{\prime} & a_{22}^{\prime}
\end{array}\right]\left[\begin{array}{l}
r \\
v
\end{array}\right]+u_{0}^{2}\left[\begin{array}{l}
b_{1}^{\prime} \\
b_{2}^{\prime}
\end{array}\right] \delta
$$

where $r$ is the yaw rate, $v$ is the sway velocity, $u_{0}$ is the cruise velocity and $\delta$ is the rudder angle. $a_{i j}^{\prime}$ and $b_{i}^{\prime}$ are constant parameters depending on the type of vessel. The transfer function from $v$ to $r$ becomes

$$
H(s)=\frac{u_{0}^{2}\left(b_{1} s+u_{0} b_{2}\right)}{\left(s+u_{0} a_{1}\right)\left(s+u_{0} a_{2}\right)}
$$

where $b_{1}, b_{2}, a_{1}$ and $a_{2}$ are parameters given by $a_{i j}^{\prime}$ and $b^{\prime}{ }_{i}$. 
Now, we generally have that $a_{1} / a_{2}$ is of the order 10-20. An acceptable approximation is therefore to assume $a_{2}=0$. Then we get the following state space representation where also the yaw angle $\psi$ is included in the model of the steering dynamics.

$$
\left.\begin{array}{l}
\dot{\psi}=r \\
\dot{r}=u_{0} a_{1} r+v+u_{0}^{2} b_{1} \delta+\text { noise } \\
\dot{v}=u_{0}{ }^{3} b_{2} \delta+\text { noise }
\end{array}\right\}
$$

where $v$ is a state variable approximately proportional to $u_{0} v$ defined in eqn. (2).

Note that a further simplification would be to introduce the Nomoto model $H(s)=K / s(1+T s)$. However, it turns out that the zero in the transfer function resulting from eqn. (3) is quite important from the control point of view. If the sway equation is totally omitted, a clear decrease in the quality of the autopilot operation is experienced. This is easily seen by simulation experiments.

\section{HF-model}

For the HF-part of the yaw motion the following model is chosen

$$
\left.\begin{array}{c}
\dot{\omega}=s+\text { noise } \\
\dot{s}=-c \omega
\end{array}\right\}
$$

where $\omega$ is the HF-part of the yaw angle, $s$ is the corresponding rate and $c$ is the square of the angular frequency of the oscillation. This model is also used for similar purposes in dynamic positioning applications (Balchen $e$ t al. 1980). The model is only neutrally stable. This, however creates no problem when used in a Kalman filter structure. Note that process noise is included only in the $\omega$-equation. This is done in order to minimize the influence of the HF-model at the low frequency end of the motion spectrum.

\section{Measurement model}

The yaw measurement model is given by

$$
y=\psi+\omega+\text { noise }
$$

\section{Estimation and control algorithms}

The adaptive autopilot is designed in discrete form. The continuous equations are discretized by the Euler method, and the sampling time is assumed to be equal to $\Delta T=1$ second.

The structure of the adaptive auto-pilot is given in Fig. 1.

A steady state Kalman filter is used as a state estimator, with gain scheduling dependent on the parameter estimates. The feedback control is taken from the LF-part of the state estimator. The adaption algorithm estimates the parameters using a prediction error based Gauss-Newton method and continuously adjusts the model parameters of the filter and the feedback control parameters.

\section{State estimator}

A Kalman filter with scheduled gains is used as a state estimator. In order to obtain integral action of the total controller a new state is introduced in the LF-part of the 


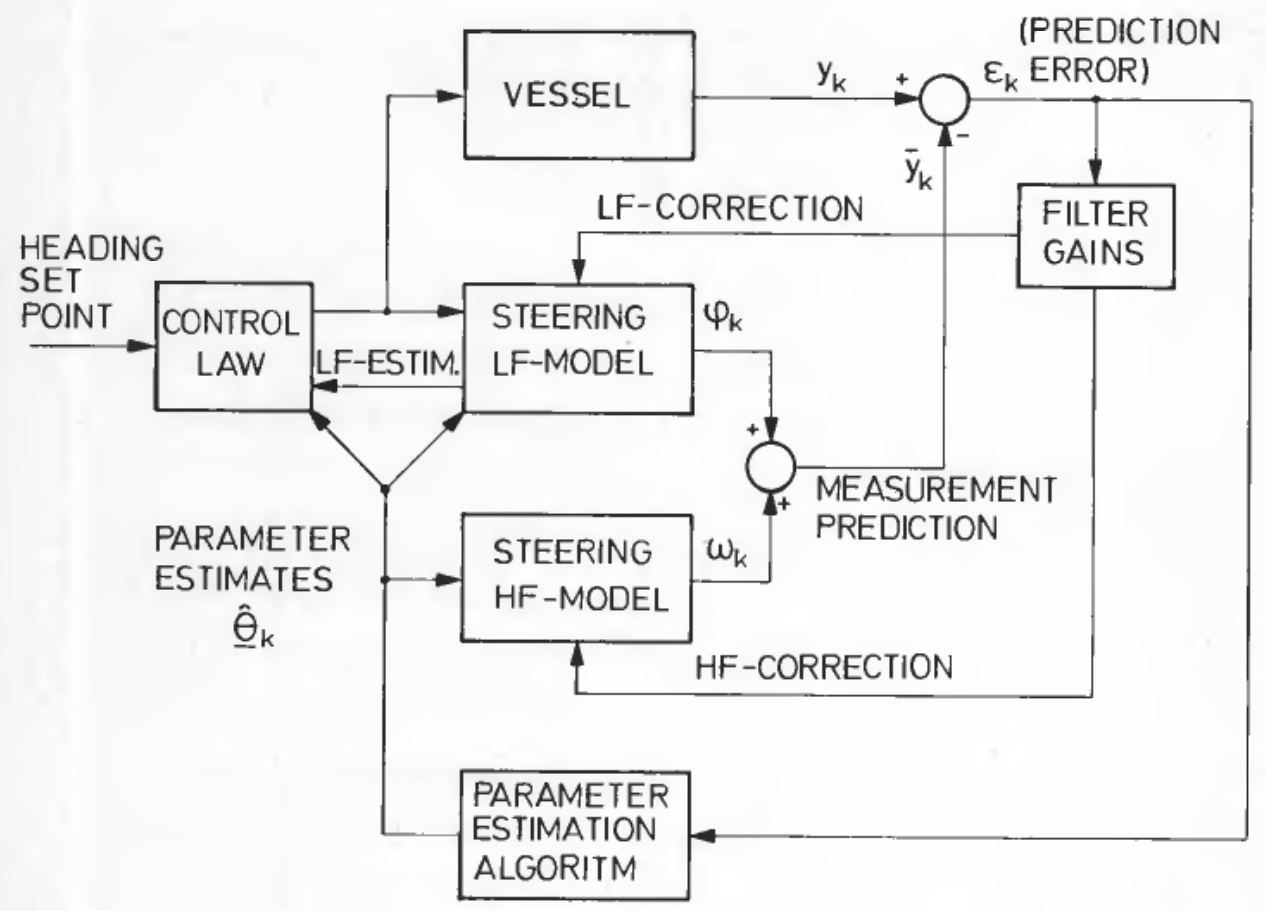

Figure 1. Adaptive autopilot structure.

model. This state accounts for unknown steady moment generating forces from wind, current etc. The discrete model becomes

$$
\left.\begin{array}{rl}
\psi_{k+1} & =\psi_{k}+r_{k} \\
r_{k+1} & =a r_{k}+v_{k}+b_{1} \delta_{k}+n_{1 k} \\
v_{k+1} & =v_{k}+p_{k}+b_{2} \delta_{k}+n_{2 k} \\
p_{k+1} & =n_{3 k} \\
\omega_{k+1} & =\cos \sqrt{ } c \cdot \omega_{k}+(\sin \sqrt{ } c) / \sqrt{ } c \cdot s_{k}+n_{k 4} \\
s_{k+1} & =-\sqrt{ } c \sin \sqrt{ } c \cdot \omega_{k}+\cos \sqrt{ } c \cdot s_{k} \\
y_{k} & =\psi_{k}+\omega_{k}+w_{k}
\end{array}\right\} \text { HF-model }
$$

where $k$ is the discrete time parameter. $p_{k}$ is the new state variable representing slowly varying current and wind generated moments and $n_{i k}, i=1,2,3,4$ are white process noise components.

Instead of the previously defined parameters we have redefined the parameters so that $a \triangleq\left(1-u_{0} a_{1}\right), b_{1} \triangleq u_{0}^{2} b_{1}$ and $b_{2} \triangleq u_{0}{ }^{3} b_{2}$. We observe that $a, b_{1}$ and $b_{2}$ depends on $u_{0}$ in a predetermined way. When known changes of $u_{0}$ are introduced the parameters $a, b_{1}$ and $b_{2}$ are changed according to these known relations. However, throughout the remaining part of this paper, $u_{0}$ is assumed to be constant. 
On vector form the total state model becomes

$$
\left.\begin{array}{c}
x_{k+1}=\phi_{0} x_{k}+B_{0} u_{k}+n_{k} \\
y_{k}=D x_{k}+w_{k}
\end{array}\right\}
$$

where $\boldsymbol{x}_{k}=\left[\psi_{k}, r_{k}, v_{k}, p_{k}, \omega_{k}, s_{k}\right]^{T}, n_{k}=\left[0, n_{1 k}, n_{2 k}, n_{3 k}, n_{4 k}, 0\right]^{T}$ and $B_{0}$ and $\phi_{0}$ are the control and transition matrices with the actual parameters inserted as defined by eqns. (6) and (7). The white noise processes are characterized by $E\left(n_{k}\right)=0, E\left(w_{k}\right)=0$, $\operatorname{cov}\left(n_{k}\right)=\operatorname{diag}\left\{0, N_{1}, N_{2}, N_{3}, N_{4}, 0\right\}$ and $\operatorname{cov}\left(w_{k}\right)=W$.

The filter equations may be written

$$
\begin{aligned}
& \overline{\boldsymbol{x}}_{k+1}=\phi \overline{\boldsymbol{x}}_{k}+B u_{k}+\phi K \epsilon_{k} \\
& y_{k}=\bar{y}_{k}+\epsilon_{k}=D \overline{\boldsymbol{x}}_{k}+\epsilon_{k} \\
& \hat{\boldsymbol{x}}_{k}=\overline{\boldsymbol{x}}_{k}+K \epsilon_{k}
\end{aligned}
$$

where $\overline{\boldsymbol{x}}_{\boldsymbol{k}}$ is the a priori estimate of $\boldsymbol{x}_{k}, \hat{\boldsymbol{x}}_{\boldsymbol{k}}$ is the a posteriori estimate of $\boldsymbol{x}_{k}, \boldsymbol{\epsilon}_{\boldsymbol{k}}$ is the prediction error, $\bar{y}_{k}$ is the measurement prediction and $\phi$ and $B$ are the transition and control matrices with the estimates of the parameters inserted.

Now the Kalman filter gain matrix $K=\left[k_{1}, k_{2}, k_{3}, k_{4}, k_{5}, k_{6}\right]^{T}$ will be independent of the parameters $b_{1}$ and $b_{2}$, but will change slightly as a function of $a$ and $c$. For realistic vessels and waves, $a$ and $c$ will certainly be in the range

$$
\left.\begin{array}{l}
a \in[0 \cdot 7,1 \cdot 0] \\
c \in[0 \cdot 1,0 \cdot 8]
\end{array}\right\}
$$

The noise parameters are chosen as $\operatorname{cov}\left(w_{k}\right)=10^{-5}(\mathrm{rad})^{2}$ and $\operatorname{cov}\left(\boldsymbol{n}_{k}\right)=\operatorname{diag}$ $\left\{0,10^{-8}, 10^{-9}, 10^{-12}, 3 \times 10^{-4}, 0\right\}$. The steady state Kalman filter gain matrix $K$ for some values of the parameters are shown in the Table. In the autopilot, the parameter space $(a, c)$ defined by eqn. (11) is discretized in a $4 \times 5$ grid and the steady state filter gain is computed for each parameter combination a priori. During operation the actual gain matrix is interpolated between the gridpoints in the two dimensional parameter space. In addition $k_{3}$ and $k_{4}$ are fairly constant so only $k_{1}, k_{2}, k_{5}$ and $k_{6}$ are interpolated. This results in a very modest computational burden.

\section{The feedback law}

As explained in the introduction, feedback is only taken from the LF-part of the state vector. The wave induced oscillatory part of the measurement will then be absorbed by the HF-model of the filter and will not be transformed into rudder action. This yields a very effective wave filtering which will later be demonstrated by simulation experiments. We shall therefore discuss the control law in relation to the LF-model.

The feedback law is chosen to be given by

$$
u_{k}=g_{1}\left(\psi_{k}-\psi_{\mathrm{ref}}\right)+g_{2} \hat{r}_{k}-\hat{v}_{k} / \hat{b}_{1}
$$

where $g_{1}$ and $g_{2}$ are feedback constants, $\psi_{\text {ref }}$ is the set point, and $\hat{b}_{1}$ is the estimate of $b_{1}$ at time $k . \hat{\psi}_{k}, \hat{r}_{k}$ and $\hat{v}_{k}$ are a posteriori estimates. The last term in eqn. (12) counteracts the influence of $v_{k}$ and $p_{k}$ and will give integral control action in the system. 


\begin{tabular}{|c|c|c|c|c|c|c|}
\hline & \multirow[b]{2}{*}{$a$} & \multicolumn{5}{|c|}{$c$} \\
\hline & & $0 \cdot 1$ & 0.2 & 0.4 & 0.6 & 0.8 \\
\hline $\begin{array}{l}k_{1} \\
k_{2} \\
k_{5} \\
k_{6}\end{array}$ & 0.7 & $\begin{array}{l}1 \cdot 44 E-2 \\
6 \cdot 49 E-3 \\
8 \cdot 29 E-1 \\
1 \cdot 17 E-1\end{array}$ & $\begin{array}{l}1 \cdot 16 \mathrm{E}-1 \\
6 \cdot 30 \mathrm{E}-3 \\
8 \cdot 57 \mathrm{E}-1 \\
1 \cdot 22 \mathrm{E}-1\end{array}$ & $\begin{array}{l}9 \cdot 77 \mathrm{E}-2 \\
6 \cdot 16 \mathrm{E}-3 \\
8 \cdot 75 \mathrm{E}-1 \\
1 \cdot 21 \mathrm{E}-1\end{array}$ & $\begin{array}{l}8 \cdot 99 \mathrm{E}-2 \\
6 \cdot 10 \mathrm{E}-3 \\
8 \cdot 83 \mathrm{E}-1 \\
1 \cdot 14 \mathrm{E}-1\end{array}$ & $\begin{array}{l}8 \cdot 53 \mathrm{E}-2 \\
6 \cdot 10 \mathrm{E}-3 \\
8 \cdot 88 \mathrm{E}-1 \\
1 \cdot 03 \mathrm{E}-1\end{array}$ \\
\hline $\begin{array}{l}k_{1} \\
k_{2} \\
k_{5} \\
k_{6}\end{array}$ & 0.8 & $\begin{array}{l}1 \cdot 80 \mathrm{E}-1 \\
9 \cdot 32 \mathrm{E}-3 \\
7 \cdot 93 \mathrm{E}-1 \\
1 \cdot 33 \mathrm{E}-1\end{array}$ & $\begin{array}{l}1 \cdot 44 \mathrm{E}-1 \\
9 \cdot 01 \mathrm{E}-3 \\
8 \cdot 30 \mathrm{E}-1 \\
1 \cdot 40 \mathrm{E}-1\end{array}$ & $\begin{array}{l}1 \cdot 19 E-1 \\
8 \cdot 76 E-3 \\
8 \cdot 55 E-1 \\
1 \cdot 41 E-1\end{array}$ & $\begin{array}{l}1 \cdot 08 \mathrm{E}-1 \\
8 \cdot 64 \mathrm{E}-3 \\
8 \cdot 64 \mathrm{E}-1 \\
1 \cdot 33 \mathrm{E}-1\end{array}$ & $\begin{array}{l}1 \cdot 02 \mathrm{E}-1 \\
8 \cdot 57 \mathrm{E}-3 \\
8 \cdot 72 \mathrm{E}-1 \\
1 \cdot 20 \mathrm{E}-1\end{array}$ \\
\hline $\begin{array}{l}k_{1} \\
k_{2} \\
k_{5} \\
k_{6}\end{array}$ & 0.9 & $\begin{array}{l}2 \cdot 50 \mathrm{E}-1 \\
1 \cdot 62 \mathrm{E}-2 \\
7 \cdot 24 \mathrm{E}-1 \\
1 \cdot 59 \mathrm{E}-1\end{array}$ & $\begin{array}{l}1 \cdot 95 \mathrm{E}-1 \\
1 \cdot 53 \mathrm{E}-2 \\
7 \cdot 80 \mathrm{E}-1 \\
1 \cdot 70 \mathrm{E}-1\end{array}$ & $\begin{array}{l}1 \cdot 56 \mathrm{E}-1 \\
1 \cdot 44 \mathrm{E}-2 \\
8 \cdot 19 \mathrm{E}-1 \\
1 \cdot 72 \mathrm{E}-1\end{array}$ & $\begin{array}{l}1 \cdot 39 \mathrm{E}-1 \\
1 \cdot 40 \mathrm{E}-2 \\
8 \cdot 36 \mathrm{E}-1 \\
1 \cdot 62 \mathrm{E}-1\end{array}$ & $\begin{array}{l}1 \cdot 29 \mathrm{E}-1 \\
1 \cdot 37 \mathrm{E}-2 \\
8 \cdot 45 \mathrm{E}-1 \\
1 \cdot 47 \mathrm{E}-1\end{array}$ \\
\hline $\begin{array}{l}k_{1} \\
k_{2} \\
k_{5} \\
k_{6}\end{array}$ & $1 \cdot 0$ & $\begin{array}{l}4 \cdot 06 \mathrm{E}-1 \\
3 \cdot 87 \mathrm{E}-2 \\
5 \cdot 70 \mathrm{E}-1 \\
2 \cdot 00 \mathrm{E}-1\end{array}$ & $\begin{array}{l}2 \cdot 94 \mathrm{E}-1 \\
3 \cdot 28 \mathrm{E}-2 \\
6 \cdot 82 \mathrm{E}-1 \\
2 \cdot 15 \mathrm{E}-1\end{array}$ & $\begin{array}{l}2 \cdot 22 \mathrm{E}-1 \\
2 \cdot 84 \mathrm{E}-2 \\
7 \cdot 55 \mathrm{E}-1 \\
2 \cdot 18 \mathrm{E}-1\end{array}$ & $\begin{array}{l}1 \cdot 92 \mathrm{E}-1 \\
2 \cdot 64 \mathrm{E}-2 \\
7 \cdot 85 \mathrm{E}-1 \\
2 \cdot 06 \mathrm{E}-1\end{array}$ & $\begin{array}{l}1 \cdot 75 \mathrm{E}-1 \\
2 \cdot 53 \mathrm{E}-2 \\
8 \cdot 02 \mathrm{E}-1 \\
1 \cdot 87 \mathrm{E}-1\end{array}$ \\
\hline
\end{tabular}

Note: $k_{3}$ is nearly constant and equal to $1 \cdot 80 \mathrm{E}-3$. $k_{4}$ is nearly constant and equal to $1 \cdot 10 \mathrm{E}-5$.

Filter gains as a function of $a$ and $c$.

As long as the parameter estimates are close to their correct value, the stability of the control depends on the eigenvalues of the matrix $\left(\phi_{\mathbf{L F}}-B_{\mathrm{LF}} G\right)$ where $\phi_{\mathrm{LF}}$ is the transition matrix for the LF-subsystem and $B_{\mathrm{LF}}$ is the corresponding control matrix. $G$ is the feedback operator. We have from eqns. (12) and (6)

$$
\left(\phi_{\mathrm{LF}}-B_{\mathrm{LF}} G\right)=\left[\begin{array}{cc:rr}
1 & 1 & 0 & 0 \\
-b_{1} g_{1} & a-b_{1} g_{2} & 0 & 0 \\
-b_{2} g_{1} & -b_{2} g_{2} & 1-\frac{b_{2}}{b_{1}} & 1 \\
0 & 0 & 0 & 1
\end{array}\right]
$$

Two of the eigenvalues are $\lambda=1$ and $\lambda=1-b_{2} / b_{1}$ respectively. $\lambda=1$ represents the integral action ( $p_{k}$ is uncontrollable) and $\lambda=1-b_{2} / b_{1}$ is due to the compensation term $-\hat{v}_{k} / \hat{b}_{1}$ in the control law. This mode will certainly be stable, because we always have that $b_{2} / b_{1} \ll 1$.

By looking at the matrix (13), we observe that the two first states are completely decloupled from the remaining part of the control. This means that $g_{1}$ and $g_{2}$ can be 
derived from optimal control theory applied to this decoupled system. We assume the following quadratic functional (see eqn. (2))

$$
J=E\left(\psi_{k}^{2}+\lambda \delta_{k}^{2}\right)
$$

In the continuous case the controller gains $g_{1}$ and $g_{2}$ are given by (14). These gains are approximately equal to the optimal gains in the discrete time case when $\Delta T=1$ second.

$$
\left.\begin{array}{l}
g_{1}=\sqrt{ }(1 / \lambda) \\
g_{2}=\sqrt{ }\left[\left(\frac{1-a}{b_{1}}\right)^{2}+\frac{2}{b_{1}} \sqrt{\frac{1}{\lambda}}\right]-\frac{1-a}{b_{1}}
\end{array}\right\}
$$

\section{Parameter estimation}

The unknown parameter vector is defined as $\theta=\left[a, c, b_{1}, b_{2}\right]^{T}$. The parameter estimation algorithm is chosen as a prediction error algorithm of the following form (see f.ex. Ljung 1981).

$$
\begin{aligned}
& \hat{\theta}_{k+1}=\hat{\theta}_{k}+\gamma_{k} R^{-1} \psi_{k} \cdot \epsilon_{k} \\
& R_{k+1}=R_{k}+\gamma_{k}\left(\psi_{k} \psi_{k}{ }^{T}-R_{k}\right)
\end{aligned}
$$

where $\hat{\boldsymbol{\theta}}_{\boldsymbol{k}}$ is the parameter estimate at time $k, \gamma_{k}$ is a positive scalar gain sequence tending to zero such that $k \cdot \gamma_{k} \rightarrow \mu, 0<\mu<\infty$ when $k \rightarrow \infty$. $\boldsymbol{\epsilon}_{k}$ is the filter prediction error, $\psi_{k}$ is an approximation to $\hat{c} \epsilon_{k} / \hat{i} \hat{\theta}$ and $R_{k}$ is an estimate of $E\left(\psi_{k} \psi_{k}{ }^{T}\right)$. In order to avoid inverting $R_{k}$ in eqn. (15) the following formulation of eqn. (16) is used where $P_{k}=\gamma_{k} \cdot R_{k}^{-1}$.

$$
P_{k+1}=\frac{1}{\beta_{k}} P_{k}\left[I-\psi_{k}\left(\psi_{k} P_{k} \psi_{k}+\beta_{k}\right)^{-1} \psi_{k} P_{k}\right]
$$

where $I$ is the unity matrix and

$$
\beta_{k}=\gamma_{k-1}\left(1-\gamma_{k}\right) / \gamma_{k}
$$

The sensitivities $\psi_{k}{ }^{i}=\hat{\epsilon} \epsilon_{k} / \hat{\theta} \hat{\theta}_{i}$ are computed by derivation of the equations for $\Delta x_{k}=x_{k}-x_{k}$ with respect to $\hat{\theta}_{i}$. We obtain from eqns. (9) and (10)

$$
\frac{\partial \Delta \boldsymbol{x}_{k+1}}{\partial \hat{\theta}_{i}}=\phi(I-K D) \frac{\partial \Delta \boldsymbol{x}_{k}}{\partial \hat{\theta}_{i}}-\frac{\partial \phi}{\partial \hat{\theta}_{i}} \hat{\boldsymbol{x}}_{k}-\frac{\partial B}{\partial \hat{\theta}_{i}} u_{k}-\phi \frac{\partial K}{\partial \hat{\theta}_{i}} \epsilon_{k}-\Delta \phi \frac{\partial x_{k}}{\partial \hat{\theta}_{i}}-\Delta B \frac{\partial u_{k}}{\partial \hat{\theta}_{i}}
$$

Now we assume that $\Delta \phi=\phi-\phi_{0}$ and $\Delta B=B-B_{0}$ are small and we neglect the two last terms of eqn. (19).

Hence we have

$$
\begin{gathered}
\frac{\partial \Delta \boldsymbol{x}_{k+1}}{\partial \hat{\theta}_{i}}=\phi(I-K D) \frac{\partial \Delta x_{k}}{\partial \hat{\theta}_{i}}-\frac{\partial \phi}{\partial \hat{\theta}_{i}} \hat{\boldsymbol{x}}_{k}-\frac{\partial B}{\partial \hat{\theta}_{i}} u_{k}-\phi \frac{\partial K}{\partial \hat{\theta}_{i}} \epsilon_{k} \\
\psi_{k}^{i}=D \cdot \frac{\partial \Delta x_{k}}{\partial \hat{\theta}_{i}}
\end{gathered}
$$

This yields 24 ordinary differential equations of a very simple structure. These sensitivity equations have to be solved recursively and together with the state estimator. The resulting $\psi_{k}{ }^{i}$ s are used for parameter estimation via the algorithm given by eqns. 
(15) and (17). $\partial K / \partial \hat{\theta}_{i}$ are computed from the precomputed values of $K$ and are approximated by constants. This is possible because $K$ is fairly close to linear in the parameters $a$ and $c$.

\section{Convergence analysis}

We want to investigate the convergence properties of the recursive parameter estimation scheme used in the autopilot algorithm. Ljung (1977 a) has shown that the stability of an associated time invariant deterministic ordinary differential equation can be used to study convergence properties of this type of recursive algorithm. In the present case this ordinary differential equation becomes

where we define

$$
\begin{aligned}
\dot{\theta} & =R^{-1} \cdot f(\theta) \\
\dot{R} & =G(\theta)-R
\end{aligned}
$$

$$
\boldsymbol{f}(\boldsymbol{\theta})=E\left[\frac{\partial \bar{\epsilon}_{\boldsymbol{k}}}{\partial \hat{\boldsymbol{\theta}}} \bar{\epsilon}_{k}\right] \text { and } G(\boldsymbol{\theta})=E\left[\frac{\partial \bar{\epsilon}_{k}}{\partial \hat{\boldsymbol{\theta}}} \frac{\partial \bar{\epsilon}_{k}}{\partial \hat{\boldsymbol{\theta}}^{T}}\right]
$$

$\overline{\boldsymbol{\epsilon}}_{k}$ is the prediction error which would result if the parameter estimate $\hat{\boldsymbol{\theta}}$ were constant and equal to a given value for all $k$. Now the theory of Ljung says that the algorithm converges if the eqns. (22) and (23) are stable. We therefore have to find expressions for $\bar{\epsilon}_{k}$ and $\partial \bar{\epsilon}_{k} / \partial \hat{\theta}$ in order to investigate the stability of the parameter estimation algorithm.

From the state estimator equations $(10 a)-(10 c)$ we obtain by $z$-transformation and some algebraic manipulations and assuming $\hat{\theta}$ is constant

or

$$
A \bar{y}_{k}=B u_{k}+C \bar{\epsilon}_{k}
$$

$$
A y_{k}=B u_{k}+(A+C) \bar{\epsilon}_{k}
$$

where $A, B$ and $C$ are polynomials in $z$ given by

$$
\begin{aligned}
& A=H_{h} \cdot H_{a} \cdot H_{i}{ }^{3} \\
& B=H_{h} \cdot H_{i}{ }^{2} \cdot b_{1}+H_{h} \cdot H_{1} \cdot b_{2} \\
& C=H_{h}\left(H_{a} \cdot H_{i}{ }^{2} k^{\prime}{ }_{1}+H_{i}{ }^{2} \cdot k^{\prime}{ }_{2}+H_{i} k^{\prime}{ }_{3}+k^{\prime}{ }_{4}\right)+H_{a} \cdot H_{i}{ }^{2}[\{(\cos \sqrt{ } c-1) \\
& \left.\left.\times H_{d}-\sin ^{2} \sqrt{ } c\right\} k^{\prime}{ }_{5}+\left\{(\cos \sqrt{ } c-1)+H_{d}\right\} \sin \sqrt{ } c \cdot k^{\prime}{ }_{6} / \sqrt{ } c\right]
\end{aligned}
$$

where $H_{h}=\left[\left(\mathrm{z}-\cos ^{2} \sqrt{ } c\right)^{2}+\sin ^{2} \sqrt{ } c\right], H_{a}=[z-a], H_{i}=[z-1]$ and $H_{d}=[z-\cos \sqrt{ } c]$. $k^{\prime}{ }_{i}$ are the elements of the modified filter gain $\phi K$. If the correct parameter vector value is given by $\theta_{0}=\left[a_{0}, c_{0}, b_{10}, b_{20}\right]^{T}$, we can express $\bar{\epsilon}_{k}$ as

$$
\bar{\epsilon}_{k}=e_{k}+\frac{1}{A+C}\left[\Delta A y_{k}-\Delta B u_{k}-(\Delta A+\Delta C) e_{k}\right]
$$

where $\Delta A=A-A_{0}, \Delta B=B-B_{0}$ and $\Delta C=C-C_{0} . A_{0}, B_{0}$ and $C_{0}$ are the polynomials corresponding to $\hat{\boldsymbol{\theta}}=\boldsymbol{\theta}_{0}$ and $e_{k}$ is the prediction error when $\hat{\boldsymbol{\theta}}=\boldsymbol{\theta}_{0}$. Now we know that eqn. (25) is equivalent to eqns. $(10 a)-(10 b)$. Because eqns. $(10 a)-(10 b)$ have the same form as eqns. (20) and (21) we get by equivalence to eqn. (25).

$$
\frac{\partial \overline{\boldsymbol{\epsilon}}_{\boldsymbol{k}}}{\partial \hat{\boldsymbol{\theta}}}=\frac{1}{A+C}\left[\frac{\partial A}{\partial \hat{\boldsymbol{\theta}}} y_{k}-\frac{\partial B}{\partial \hat{\boldsymbol{\theta}}} u_{k}-\left(\frac{\partial A}{\partial \hat{\boldsymbol{\theta}}}+\frac{\partial C}{\partial \hat{\boldsymbol{\theta}}}\right)\right] \overline{\boldsymbol{\epsilon}}_{\boldsymbol{k}}
$$



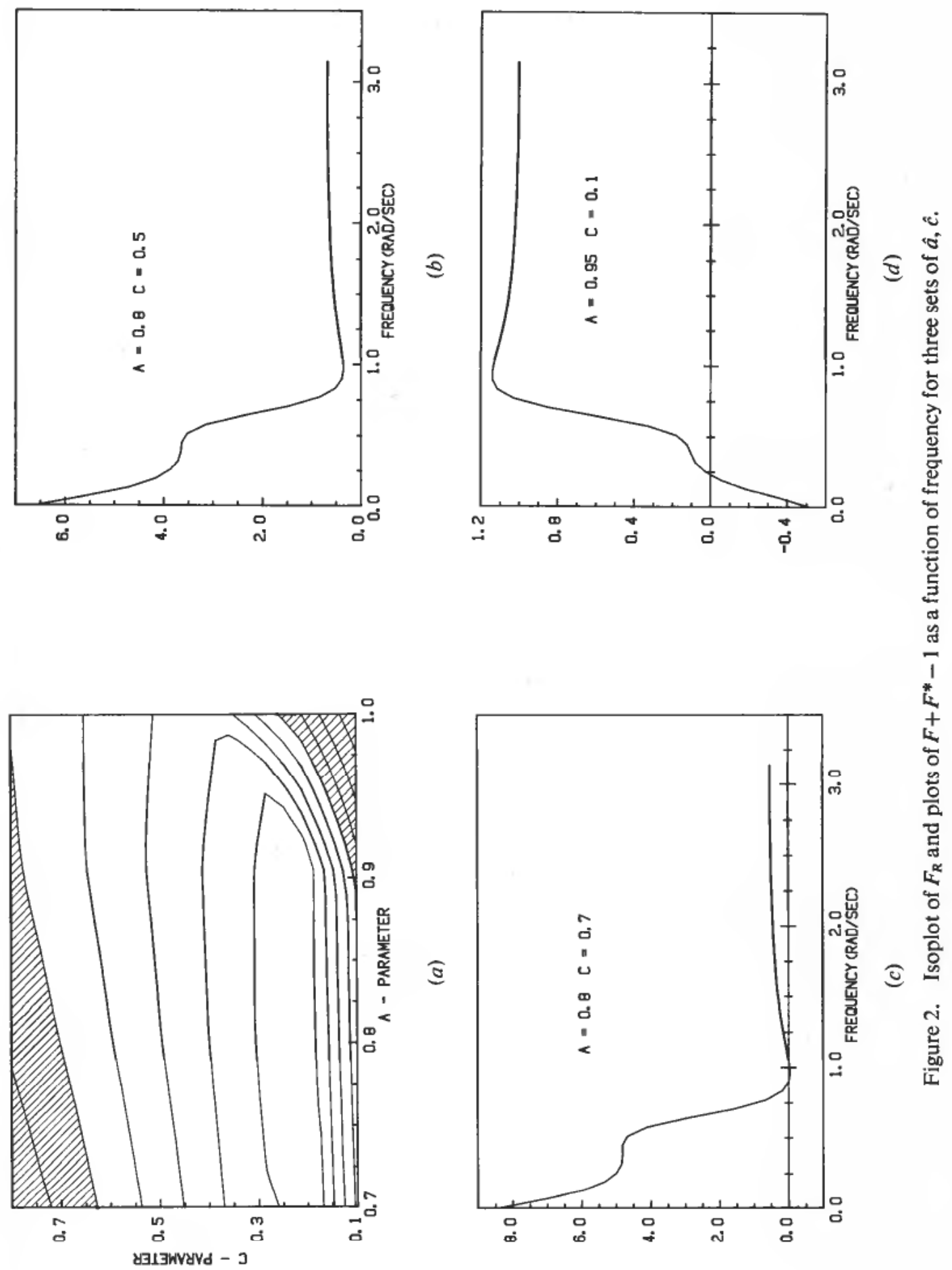

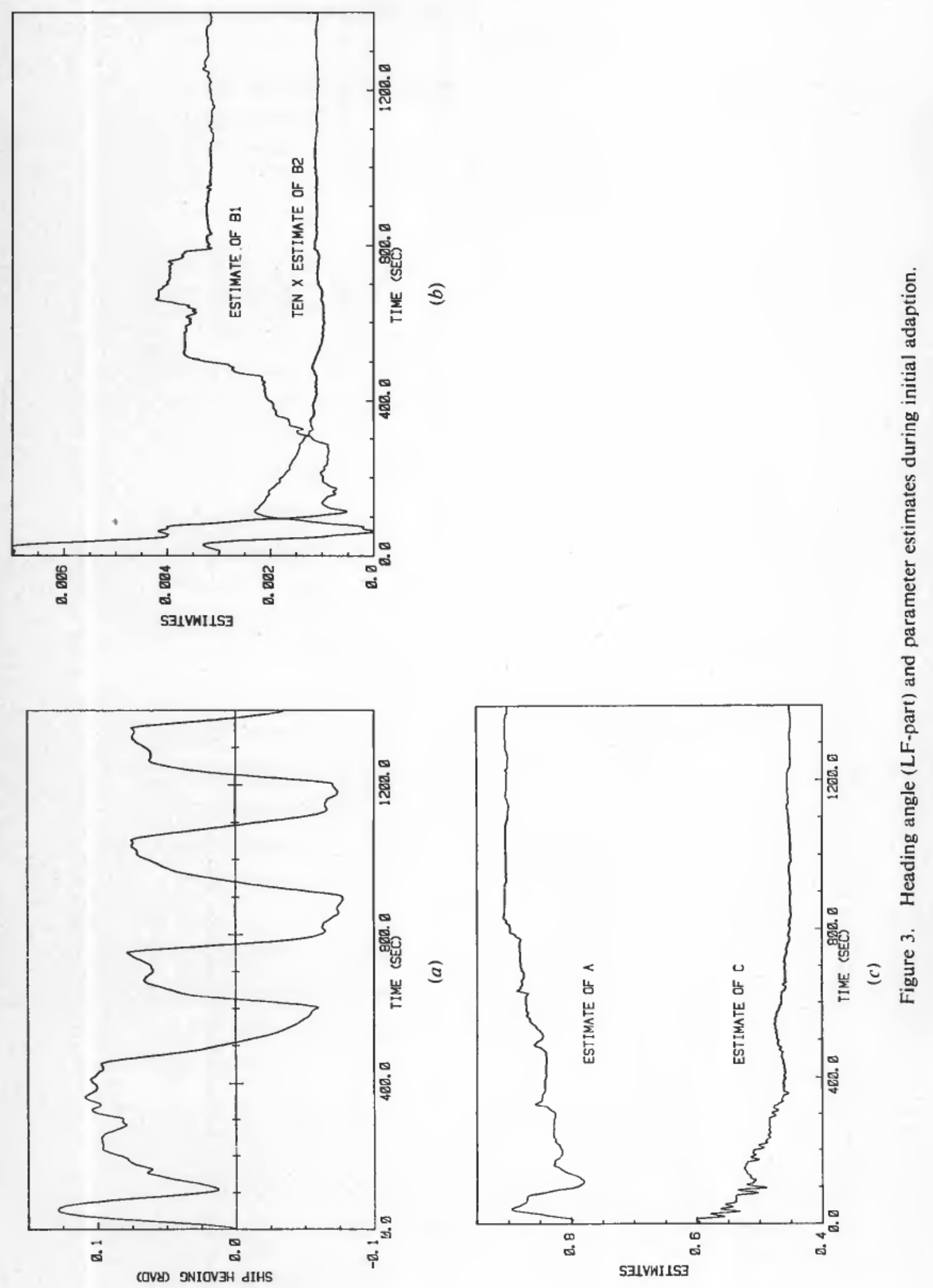

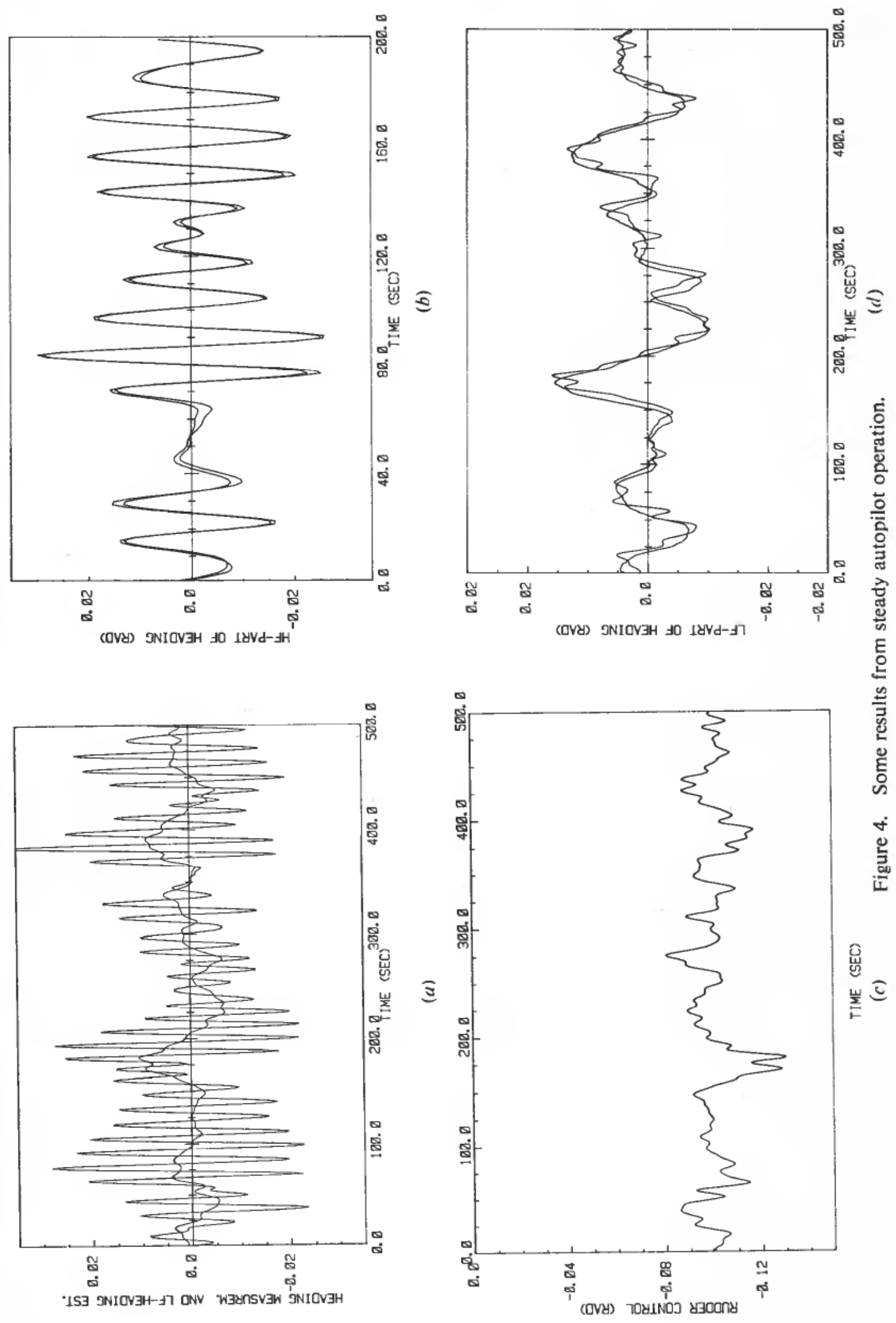
By using the fact that $\Delta B=(\hat{\partial} B / \hat{c} \theta) \cdot \Delta \theta$ and that $\Delta A \approx(\hat{\partial} A / \hat{c} \theta) \cdot \Delta \theta$, $\Delta C \approx(\partial C / \partial \theta) \cdot \Delta \theta, \Delta \theta=\hat{\theta}-\theta_{0}$ and comparing eqns. (26) and (27) we obtain the following relationship

$$
\bar{\epsilon}_{k}=e_{k}+\frac{A+C}{A_{0}+C_{0}} \frac{\partial \bar{\epsilon}_{k}}{\partial \theta^{T}}
$$

From Ljungs work (1977 b), it is then clear that the parameter estimator is stable if the transfer function

$$
F=\frac{A+C}{A_{0}+C_{0}}-\frac{1}{2}
$$

is positive real.

In order to investigate the stability properties of the algorithm, we have assumed that $a_{0}=0.9, c_{0}=0.2$ and computed the minimum value of $\left(F+F^{*}-1\right)$. An isoplot of $F_{R}=\min \left\{\left(F+F^{*}-1\right) ;|z|=1\right\}$ is shown in Fig. $2(a)$ for different values of $\hat{a}$ and $\hat{c}$. The non-positive area is indicated as the hatched part of the plot. We observe that the algorithm is stable for a broad range of $\hat{a}$ and $\hat{c}$. The unstable combinations occur at $\hat{c}$ small, $\hat{a}$ large and for $\hat{c}$ large, $\hat{a}$ small. The estimator should therefore prevent $\hat{a}$ and $\hat{c}$ from entering these domains. Three plots of $F_{R}$ as a function of frequency are also shown in Fig. $2(b-d)$. For $\hat{a}=1, \hat{c}=0 \cdot 1$, the instability occurs in the low frequency domain. For $\hat{a}=0 \cdot 8, \hat{c}=0 \cdot 7$, the instability occurs in a higher frequency domain which is quite natural.

\section{Simulation experiments}

In this section we shall give some of the simulation results where the control system is working against a computer implemented simulator. The simulator includes realistic wave excitations in yaw, unknown steady moments from wind and current and white process noise excitations of the sway- and yaw-rate equations. The dynamic relations between $r$ and $v$ are simulated by eqn. (2). The coefficients are taken from Källström (1979) and represents a cargo ship of the Mariner class. The coefficients of eqn. (2) are given as

$$
\begin{array}{ccc}
u_{0} a_{11}^{\prime}=-0.036 ; & u_{0} a_{12}^{\prime}=-0.25 ; & u_{0} a_{21}^{\prime}=-0.00099 ; \quad u_{0} a_{22}^{\prime}=-0.098 ; \\
u_{0}^{2} b_{1}=0.060 ; & u_{0}^{2} b_{2}^{\prime}=-0.0036 .
\end{array}
$$

During the initial adaptive period (for approx. 20 minutes) the heading reference is cycled with a period of 5 minutes and an amplitude of $4^{\circ}$. The actual ship heading is shown in Fig. $3 a$ ). The control is very bad during the first 5-8 minutes until the parameters have converged. The parameters converge in 10 to 15 minutes. Comparing the parameter estimates to the equivalent simulation model parameters, the estimates are correctly estimated within roughly $+10 \%$ of actual values.

In order to prevent the parameter updating gains to go to zero when $k \rightarrow \infty, \beta_{k}$ in eqn. (18) is set to $0 \cdot 9975$. The steady state operation of the autopilot is illustrated in Fig. 4. Figure $4(a)$ shows the heading measurement and the LF-estimatc of the heading. As is seen, a very good wave filtering of the heading measurement is obtained. The actual HF-part of the heading (simulator HF-part) and the estimate of the HF-part is shown in Fig. $4(b)$ for the section from $t=300$ to $t=500$ of Fig. $4(a)$. The rudder control corresponding to Fig. $4(a)$ is shown in Fig. $4(c)$. The actual (simulated) and estimated LF-part of the heading is shown in Fig. $4(d)$. 


\section{Conclusions}

An adaptive autopilot based on a steering model of a ship with six states and four physically related parameters is designed and tested against a simulator. The adaption is based on a Kalman filter with gain scheduling and an error prediction based parameter estimation algorithm. A special feature of the autopilot is a model of the wave induced yaw motion which effectively reduces the wave excitation of the rudder control. The convergence properties of the autopilot are analysed by Ljung's method and the effective operation of the system is confirmed by simulation experiments.

\section{REFERENCES}

Åstrom, K. J., and Källström, C. G. (1976). Identification of ship steering dynamics. Automatica., 12, 9.

Balchen, J. G., Jenssen, N. A., and Salid, S. (1980). Dynamic positioning of floating vessels based on Kalman filtering and optimal control theory. Proceedings 19th I.E.E.E. Conference on Decision and Control, Albuquerque, USA, pp. 852-864.

KÄLlströM, C. G. (1979). Identification and adaptive control applied to ship steering. Thesis, Dept. of Automatic Control, Lund Institute of Technology, Lund, Sweden.

Källström, C. G., Åström, K. J., Thorell, N. E., Erikson, J., and Sten, L. (1979). Adaptive autopilots for tankers. Automatica, 15, 241-254.

KÄllström, C. G., and Åstrom, K. J. (1981). Experiences of systems identification applied to ship steering. Automatica, 17, 1, 187-198.

LJUNG, L. (1977 a). Analysis of recursive stochastic algorithms. I.E.E.E. Trans., AC-22, 551-575.

LJUNG, L. (1977 b). On positive real transfer functions and the convergence of some recursive schemes. I.E.E.E. Trans., AC-22, 539-551.

LJUNG, L. (1981). Analysis of a general recursive prediction error identification algorithm. Automatica, 71, 1, 89-99.

Norrbin, N. H. (1970). Theory and observations on the use of a mathematical model for ship manoeuvering in deep and confined waters. Proceedings 8 th Symposium on Naval Hydrodynamics. Pasadena, California.

Norrbin, N. H. (1972). On the added resistance due to steering on a straight course. 13th International Towing Tank Conference, Berlin/Hamburg.

Ohtsu, K., Horigome, M., and Kitagawa, G. (1979). A new ship's auto pilot design through a stochastic model. Automatica, 15, 3, 255-268. 